\title{
Akademisches Schreiben lehren und lernen - Spektren einer prozessorientierten Schreibdidaktik
}

\section{Dagmar Knorr \& Melanie Brinkschulte}

Journal für Psychologie, 27(1), 51-71

https://doi.org/10.30820/0942-2285-2019-1-51

www.journal-fuer-psychologie.de

\section{Zusammenfassung}

Von Studierenden wird erwartet, dass sie akademische Texte verfassen können, die fachlichen und sprachlichen Anforderungen gerecht werden. Der Umgang mit fachlichen und textuellen Konventionen sowie das Schreibhandeln der Studierenden sind wichtige Bestandteile der Arbeit von Schreibzentren. Gemeinsam mit Lehrenden werden Anforderungen an Texte herausgearbeitet. Zudem werden die Anforderungen an Texte untersucht, die Lehrende an Texte stellen. Denn je besser fachliche und sprachliche Anforderungen an Texte beschrieben werden können, desto eher können diese vermittelt werden. Schreiben im Studium wird in drei Dimensionen beschrieben: das Nutzen des Schreibens für das kritische Denken, die produktive Steuerung des eigenen Schreibprozesses sowie die Kenntnis von Textkonventionen zur angemessenen sprachlichen Realisierung. Anhand von Beispielen wird gezeigt, wie die Arbeit von Schreibzentren sowohl fachübergreifend als auch in den Fächern selbst gestaltet werden kann. Die Kooperation mit Fachlehrenden ist ein wichtiger Baustein. Hinzu kommt die Arbeit mit Studierenden und die Ausbildung von Studierenden zu studentischen Schreibberater_innen und Writing Fellows. Es wird gezeigt, wie hieraus Nutzen für die gesamte Hochschule entstehen kann. Abschließend wird die derzeitige Stellung von Schreibzentren in der Hochschullandschaft kritisch beleuchtet.

Schlüsselwörter: Schreibdidaktik, wissenschaftliches Schreiben, Schreibwissenschaft, Hochschulentwicklung, Schreibkompetenz

\section{Summary}

Teaching and Learning Academic Writing - principles of a process-oriented writing pedagogy

Instructors expect students to submit academic texts that do not contain any traces of the writing process and that conform to disciplinary and stylistic conventions. These conventions and the practices of student writers are at the core of writing center work. We work closely with faculty staff to understand professors' expectations concerning student work in 
particular and academic writing in general. The results of this collaborative effort can be used to improve the quality of instruction in and outside of the classroom, to develop new instructional formats, or to provide high-quality feedback on disciplinary writing. Here, we discuss three dimensions of this kind of writing: writing as a means to develop critical thinking and facilitate active learning; an understanding of writing processes and the ability to design the processes; and an understanding of conventions and the ability to apply this kind of knowledge. Discussing several successful approaches taken at German writing centers, we show that it is possible to work both across and within disciplines. Key elements are a close cooperation with faculty, individual consultations and workshops and peer tutor and writing fellows programs. All of these programs can, we suggest, be beneficial to universities. We conclude by discussing the potential contributions of writing centers to higher education.

Keywords: writing center work, academic writing, writing studies, strategic development, writing competences

\section{Einleitung}

Schreiben ist ein wesentlicher Bestandteil wissenschaftlichen Arbeitens und Denkens. Schreibend können Inhalte erarbeitet werden und somit trägt Schreiben zum Lernen bei; Ergebnisse von Lern- und Arbeitsprozessen werden wiederum schriftlich festgehalten und können auf diese Weise Leser_innen weitergegeben werden. Schreiben hat also eine doppelte Funktion: Es ist Denkwerkzeug und Präsentationsmedium. Für Schreibdidaktiker_innen und Schreibwissenschaftler_innen ist Schreiben zudem der Gegenstand ihrer Lehre und Forschung. Sie untersuchen das Schreiben, als wissenschaftlich zu untersuchenden Gegenstand, sie interagieren mit Personen und gehen in dieser Arbeit auf die individuellen Schreibpraxen ein, und sie reflektieren über die Art und Weise, wie sie das Schreiben und Schreibprodukte betrachten, um das eigene Handeln zu kontrollieren (vgl. Kruse und Ruhmann 2006).

Schreiben ist zudem eine situierte Handlung (Ehlich 2003). Hochschulen sind Orte, an denen schriftsprachliche Konventionen gepflegt werden und deren Einhaltung von Studierenden erwartet wird. Dies zeigt sich unter anderem in den verschiedenen Formaten schriftlicher Prüfungsleistungen.

Die Konventionen sind nicht nur durch die Hochschule selbst, sondern auch fachspezifisch, kulturell, sprachlich und zeitlich geprägt (Jakobs 1997). Studierende haben die Aufgabe, sich diese Konventionen im Verlauf ihres Studiums anzueignen. Hierbei hat die Fachlehre einen entscheidenden Anteil, die jedoch zu einem großen Anteil mündlich geprägt ist. In Veranstaltungsformen wie der Vorlesung und dem Seminar stehen mündliche Kommunikationen im Vordergrund. Die Überprüfung des Aneignungsprozesses erfolgt 
dann in den meisten Fächern auf der Basis eines schriftlichen Produkts. Das schriftliche Produkt, der akademische Text, kann sehr unterschiedliche Formen annehmen: So gehören Protokolle, Essays, Portfolios oder Haus- oder Seminararbeiten zu den akademischen Textarten (vgl. Ehlich 2003). Unabhängig von der abzuliefernden Textform wird geprüft und bewertet, inwieweit der abgelieferte Text der Anforderung entspricht, eine Aufgabenstellung inhaltlich und sprachlich angemessen und mit wissenschaftlichen Methoden bearbeitet zu haben. Anders ausgedrückt: Es wird neben der fachlichen Einordnung geprüft, inwieweit Konventionen der Disziplin umgesetzt werden konnten:

$>$ Konventionen des Faches: Wahl und Ausführung der Methode;

$>$ Konventionen des Textmusters: Aufbau und Gliederung des Textes;

$>$ Konventionen der sprachlichen Realisierung: angemessener schriftsprachlicher Sprachgebrauch, der sich unter anderem in der Verwendung fachlicher Ausdrücke, aber auch in der Verbindung dieser Ausdrücke, also der Verwendung »alltäglicher Wissenschaftssprache « (Ehlich 1999) zeigt.

Ob der Prozess, der zu dem Produkt geführt hat, in die Bewertung einbezogen wird oder nicht, obliegt der Verantwortung der Lehrenden. Eine wichtige Rolle spielen hierbei die zu erbringenden Prüfungsleistungen: Während bei Hausarbeiten der Prozess in der Regel nicht berücksichtigt wird, eröffnet die Arbeit mit Portfolios entsprechende Möglichkeiten (vgl. G. Bräuer 2016). Inwieweit prozessuale Aspekte in die Bewertung von Texten eingehen, stellt ein Forschungsdesiderat der Schreibwissenschaft dar.

Im Folgenden möchten wir den Prozess des Entstehens von Textprodukten in den Fokus rücken und zeigen, welche Aspekte eine Schreibausbildung unseres Erachtens im Studium aufgreifen sollte. Denn der Aufbau wissenschaftlicher Schreibkompetenz ist ein länger dauernder Prozess, der eine bewusste Auseinandersetzung und Übung erfordert (vgl. Steinhoff 2007a). Studierende sind oftmals unsicher, wie sie ihre Schreibprozesse gestalten können, um komplexe Qualifikationstexte, wie zum Beispiel Hausarbeiten, zu verfassen. In Schreibberatungen und Lehrveranstaltungen zum wissenschaftlichen Schreiben greifen Schreibdidaktiker_innen diese Unsicherheiten, Lernbedarfe, aber auch vorhandene Ressourcen von Studierenden auf, um ihnen individualisierte und zielorientierte Handlungswege aufzuzeigen (vgl. Alagöz-Bakan, Knorr und Krüsemann 2016; Ballweg 2016; Brinkschulte 2016).

Auch Lehrende sehen zunehmend die Notwendigkeit, Studierende im wissenschaftlichen Schreiben auszubilden, sei es, dass sie es in den zu bewertenden Texten bemängeln oder wenig Engagement aufseiten der Studierenden wahrnehmen, beispielsweise wenn es um das Wahrnehmen von Betreuungsangeboten oder Hausarbeitsbesprechungen geht. In der schreibdidaktischen Begleitung von Lehrenden entwerfen Schreibdidaktiker_innen gemeinsam mit den Lehrenden Konzepte zur Integration von Schreiben als Lerngegenstand in der Lehre (vgl. bspw. Böhner et al. 2016). 
Im Folgenden werden wir zentrale Inhalte einer prozessorientierten Schreibdidaktik skizzieren. Im nächsten Abschnitt werden anhand eines Beispiels typische Merkmale eines wissenschaftlichen Textes illustriert. Im dritten Abschnitt werden die drei Dimensionen wissenschaftlicher Schreibkompetenz näher erläutert: das Nutzen des Schreibens für das kritische Denken, die produktive Steuerung des eigenen Schreibprozesses sowie die Kenntnis von Textkonventionen zur angemessen sprachlichen Realisierung. Im vierten Abschnitt legen wir dar, wie Schreibzentren die Schreibentwicklung von Studierenden unterstützen, indem wir Tätigkeitsfelder von Schreibzentren beschreiben. Hieraus leiten wir die Frage ab, welchen Nutzen Fakultäten, Fachbereiche, Institute, Lehrende und Studierende aus der Arbeit mit Schreibdidaktiker_innen ziehen können und wie Schreibdidaktik als Querschnittsaufgabe von Hochschulen institutionalisiert werden kann.

\section{Vom Produkt zum Prozess - Schreibprozesse untersuchen}

Studentische Texte können als »Lernformen « (Pohl und Steinhoff 2010) betrachtet werden, die den Stand des Erwerbs wissenschaftlicher Schreibkompetenz widerspiegeln. Im schreibwissenschaftlichen Diskurs hat sich für die Beschreibung studentischer Textproduktionen der Begriff » akademisches Schreiben « (Jakobs 1999) durchgesetzt. Akademisches Schreiben bedarf zwar ebenfalls einer an wissenschaftssprachlichen Konventionen orientierten Sprache, jedoch wird von einem studentischen Text beispielsweise nicht erwartet, dass in ihm neues Wissen präsentiert wird. Auch kann - zumindest in den ersten Jahren des Studiums - nicht davon ausgegangen werden, dass die Diskursgemeinschaft bekannt ist, in dem der Text mit seinem Ansatz verortet werden müsste. Was jedoch erwartet wird, ist die Verortung des eigenen Textes im Kontext einer wissenschaftlichen Diskussion, eines Diskurses. Die Verknüpfung der eigenen Gedanken mit in der Forschungsliteratur vorhandenen ist ein wesentlicher Bestandteil jedes wissenschaftlichen und auch jedes akademischen Textes. In solchen Punkten zeigen sich Unterschiede zwischen wissenschaftlichen Texten, die beispielsweise in Zeitschriften publiziert sind, und studentischen Texten (vgl. Graefen 1997; Stezano Cotelo 2011).

Inwieweit die Annäherung an die für wissenschaftliche Texte geforderten Kriterien gelingt, zeigt sich unter anderem in der jeweiligen kommunikativen Angemessenheit (vgl. Steinhoff 2007a). Angemessenheit im Bereich der Darstellung von Forschungspositionen zeigt sich besonders darin, wie auf Fachtexte Bezug genommen wird. Das Zitieren, Paraphrasieren und Verweisen auf Fachliteratur gehört damit zu den wichtigsten Kriterien »guter wissenschaftlicher Praxis « (Deutsche Forschungsgemeinschaft 2013), da hier die Art des Umgangs mit Wissen von anderen deutlich wird.

Was dies im Einzelnen bedeutet, soll an einem Beispiel aus einer Masterarbeit illustriert werden (vgl. Abb. 1). 

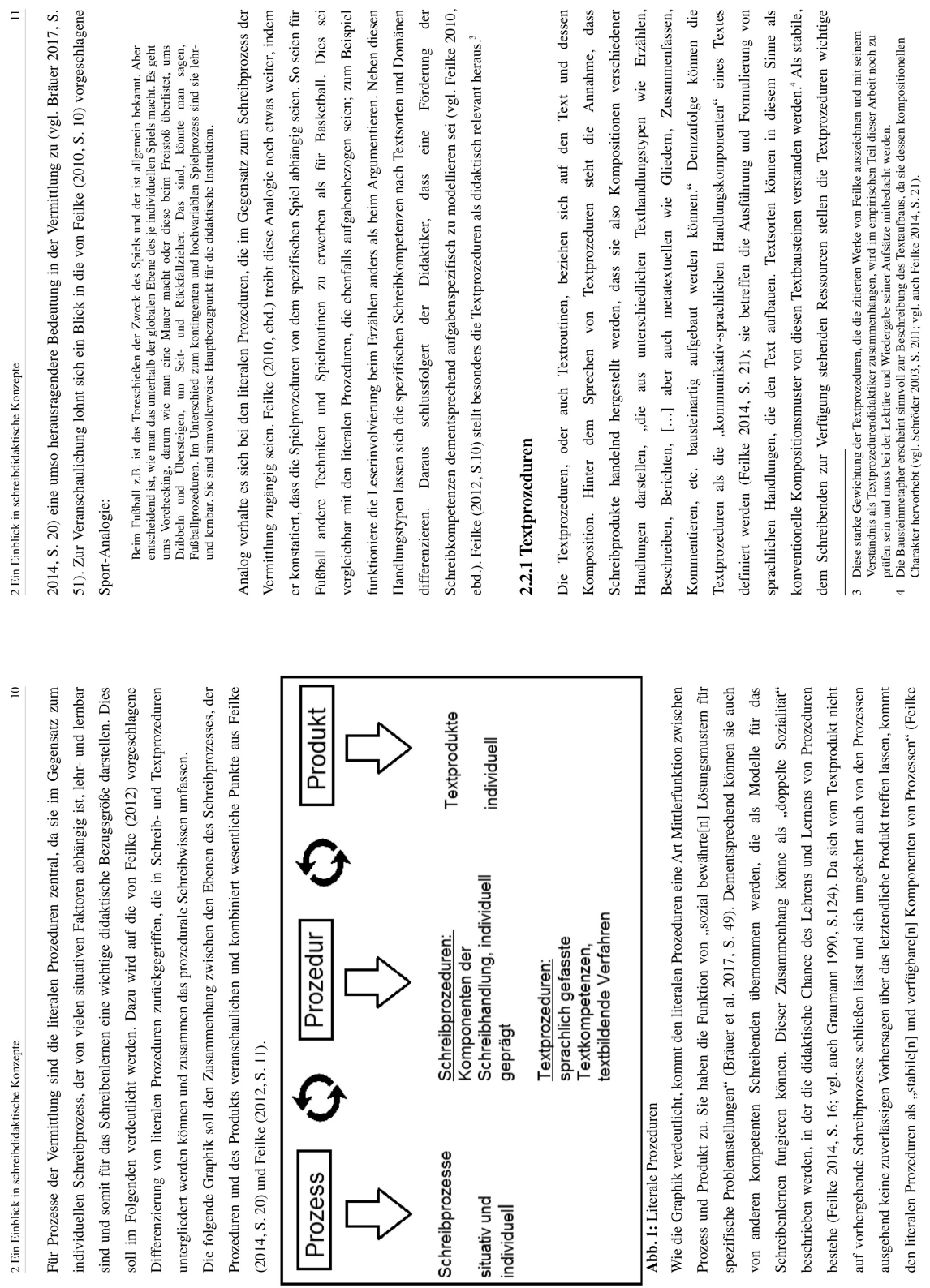

Abbildung 1: Ausschnitt aus einer Masterarbeit 
Der Ausschnitt erlaubt die Bestimmung von Merkmalen eines akademischen Textes: Es wird auf andere Fachtexte Bezug genommen, indem zitiert, paraphrasiert und verwiesen wird, in Fußnoten finden sich Anmerkungen und der Text ist sichtbar gegliedert sowie formal mit einem einheitlichen Satzspiegel gestaltet. Des Weiteren wird eine Abbildung verwendet, auf die im Text sprachlich verwiesen wird.

Als Schreibwissenschaftlerinnen interessiert uns neben dem Schreibprozess mit seinen Handlungsphasen auch, was auf sprachlicher Ebene geschieht, damit ein Text für eine_n Leser_in nachvollziehbar wird. Das bedeutet, wir schauen beispielsweise, auf welche Art und Weise sprachliche Bezüge realisiert werden und ob und wie die eigene Position deutlich wird.

Der gewählte Ausschnitt beginnt mit »Für Prozesse der Vermittlung sind die literalen Prozeduren zentral «. Hier positioniert sich die Autorin als Forscherin, indem sie die Relevanz eines vorhandenen Ansatzes für ihre eigene Arbeit als »zentral « markiert. Die Begründung liefert der nachfolgende Nebensatz: »da sie [...] lehr- und lernbar sind und somit für das Schreibenlernen eine wichtige didaktische Bezugsgröße darstellen «. Der nächste Satz dient der Leser_innenführung: »Dies soll im Folgenden verdeutlicht werden.« Und schließlich verortet sich die Autorin, indem sie ihren Ansatz an einen vorhandenen anbindet: $\gg$ Dazu wird auf die von Feilke (2012) vorgeschlagene Differenzierung von literalen Prozeduren zurückgegriffen. «Im Text selbst erfolgt dann die Darstellung des Ansatzes von Feilke sowie die Auseinandersetzung mit ihm unter Einbeziehung weiterer Literatur.

An diesem kurzen Ausschnitt wird Folgendes deutlich: Die Schreiberin versteht es, ihre eigenen Gedanken mit vorhandenen in Beziehung zu setzen und dies sprachlich angemessen zu realisieren. Doch wie ist sie zu ihrem Text gekommen? Welche Strategien hat sie verfolgt, um ihre Gedanken zu entwickeln? Welche sprachlichen und fachlichen Aspekte spielen eine Rolle? In welcher Form hat sie Gelesenes aus den zugrunde liegenden wissenschaftlichen Publikationen aufgearbeitet? Fiel es ihr leicht, die Gedanken in dieser sprachlichen Form zu produzieren oder schwer? Wie vieler Überarbeitungen bedurfte es, um zu diesem Produkt zu gelangen? Auf all diese Fragen gibt der Text keine Antwort, weil der komplexe Schreibprozess mit all seinen Arbeitsprozessen nicht im Text sichtbar ist und die Spuren der Entstehung von einer ersten Rohfassung bis zur Endversion getilgt sind. Krings $(1992,48)$ bezeichnet dies als »Produkt-Prozess-Ambiguität «: »Produkte sagen wenig oder gar nichts darüber aus, welche Prozesse ihnen jeweils vorausgegangen sind. «1

Die Schreibwissenschaft fragt unter anderem nach der Verbindung zwischen Prozess und Produkt. Wir möchten mehr darüber wissen, welche Kompetenzen notwendig sind, um zu einem guten Produkt zu gelangen, und wie man diese Kompetenzen vermitteln kann. Schreibwissenschaft legt Kriterien für gute Texte und für zugrunde liegende Schreibprozesse - also bewusst auszuwählende Handlungsmöglichkeiten - offen, sodass sie Vermittlungsprozessen zugänglich werden. 
Betrachtet man Schreiben kognitionspsychologisch, so ist eine in der Schreibwissenschaft zentrale Forschung zum Schreiben als Problemlösen von Hayes und Flower (1980) durchgeführt worden. Ihr Verdienst ist es, dass der komplexe Arbeitsprozess beim Schreiben sichtbar gemacht wird, der durch das Individuum in einem Zusammenspiel von Arbeits- und Langzeitgedächtnis und dem mentalen Monitor bewältigt werden muss. Kognitiv orientierte Modellierungen des Schreibprozesses nehmen daher bei ihren empirischen Untersuchungen von Schreibprozessen auf kognitionspsychologische Erkenntnisse Bezug (vgl. Flower und Hayes 1980; Hayes 2012). Schreibprozesse empirisch zu untersuchen, beinhaltet methodische Herausforderungen, die in den letzten 30 Jahren auch im deutschsprachigen Raum intensiv diskutiert wurden (vgl. BeckerMrotzek, Grabowski und Steinhoff 2016; Brinkschulte und Kreitz 2017; Jakobs und Perrin 2014; Knorr, Heine und Engberg 2014). Es hat sich eine Methodenvielfalt entwickelt, die es ermöglicht, entsprechend der Fragestellung gezielt Teilkomponenten des Schreibprozesses zu untersuchen oder Gesamtverläufe abzubilden und zu analysieren. Schreibdidaktik nutzt diese Erkenntnisse empirischer Schreibforschung (vgl. 3.1).

\section{Schreibkompetenz im Studium erwerben}

Wissenschaftlich zu schreiben, beinhaltet eine Reihe von Anforderungen. Wir definieren Schreibkompetenz, die im Studium erworben werden soll,

»als Fähigkeit, Texte zum Lernen und als Anknüpfungspunkte für eigene Textproduktionen zu nutzen und sich schriftsprachlich angemessen auszudrücken. Diese Fähigkeit setzt sich aus fachübergreifenden und fachspezifischen Komponenten zusammen und kann in drei Dimensionen beschrieben werden: Kompetente Schreibende nutzen Schreiben zum kritischen Denken, steuern produktiv den eigenen Schreibprozess und kommunizieren entsprechend den Textkonventionen der jeweiligen Fachgemeinschaft « (Gesellschaft für Schreibdidaktik und Schreibforschung 2018).

In diese Definition sind Erkenntnisse aus der Schreibentwicklungsforschung, Schreibdidaktik, Textlinguistik und Kognitionspsychologie eingeflossen. Im Zentrum stehen die Schreibenden selbst und ihr Umgang mit Texten. Texte werden rezipiert und produziert. Die Verbindung von Lese- und Schreibprozessen ist der Definition von Schreibkompetenz inhärent. Texte lesen und verstehen zu können, ist Voraussetzung für wissenschaftliche Schreibkompetenz. Daher spielt auch das Lesen in der Schreibzentrumsarbeit stets eine wichtige Rolle.

Die Definition der Schreibkompetenz umfasst drei Dimensionen, die auf der Basis des vorhandenen, aber auch weiterzuentwickelnden sprachlichen Wissens betrachtet 
werden müssen. Das sprachliche Wissen bildet die Ressource, auf die beim Schreiben und Lesen zurückgegriffen wird (Kruse und Jakobs 1999, 22). Je umfangreicher das sprachliche Wissen ist, desto besser kann es gelingen, Angemessenheit im wissenschaftlichen Sprachgebrauch herzustellen. Im Umkehrschluss bedeutet dies: Je geringer das sprachliche Wissen ist, desto eher stellt es eine Einschränkung beim Schreiben dar (Portmann-Tselikas 1991, 427). Schreibzentren wie das in Göttingen und Lüneburg unterstützen Studierende daher nicht nur im Erwerb komplexer akademischer Schreibkompetenzen in der Erstsprache, sondern befähigen Studierende im fremd- und zweitsprachlichen Schreiben. Zudem fördern sie Studierende, ihre individuelle Mehrsprachigkeit für das akademische Schreiben situationsangemessen und zielorientiert einzusetzen und die Wissenschaftssprachen - zumeist Deutsch und Englisch - sinnvoll in den Arbeitsprozess zu integrieren (vgl. Barczaitis, Brinkschulte und Grieshammer 2017; Brinkschulte 2012; Knorr et al. 2015; Lange 2015).

\section{Das Schreiben zum kritischen Denken nutzen}

Die Kultusministerkonferenz (2017) verfolgt für hochschulische Bildung unter anderem das Ziel, Studierende zum eigenständigen und kritischen Denken und Handeln zu befähigen, das sie während ihres Studiums erwerben und welches sie als Absolvent_innen in Beruf und Gesellschaft nutzen können sollen. Es handelt sich um eine »geistige Haltung «, die »vor das Urteil eine genaue Reflexion setzt « (Kruse 2017, 10). Es geht also um eine rationale, sorgfältige Art des Umgangs mit Wissen und Unwissen, bei der Fakten und Positionen abgewogen und hinterfragt werden. Kritisch Denkende legen ihre Argumentationen einer Wissensgemeinschaft zur Prüfung vor und treten in eine Diskussion ein. Wenn sich in der Diskussion ergibt, dass die eigene Position nicht haltbar ist, ist eine kritisch denkende Person in der Lage, diese zu revidieren beziehungsweise zu differenzieren.

Wir gehen davon aus, dass sich das Schreiben für die Förderung kritischen Denkens in besonderer Weise eignet, zumal es in allen Fächern eingesetzt werden kann, um disziplinspezifische Denkweisen zu fördern (vgl. Gesellschaft für Schreibdidaktik und Schreibforschung 2018). Das Lesen spielt für die Entwicklung kritischen Denkens im Fach eine wichtige Rolle, da über das Rezipieren von Fachtexten ebenfalls ein Zugang zu Denkweisen im Fach erfolgt, deren Inhalte zunächst nachvollzogen und zunehmend kritisch diskutiert werden können (mündlich und schriftlich). Empirische Wissenschaften, wie zum Beispiel die Psychologie, erfordern von Studierenden eine systematische, kritische Auseinandersetzung mit Daten, die es mit geeigneten Methoden auszuwerten gilt. In Texten manifestieren Studierende ihre Ergebnisse kritischen Denkens. Das Schreiben können Studierende nutzen, um kritisches Denken zu praktizieren. Dabei können Schreibtechni- 
ken, wie das Freewriting oder das Mehr-Versionen-Schreiben, die Schreibenden in ihrer kritischen Auseinandersetzung mit fachwissenschaftlichen Inhalten unterstützen.

Hochschulische Schreibaktivitäten können im Fachstudium vielfältig als Lerngelegenheiten genutzt werden: So ist beispielsweise das Mitschreiben in Lehrveranstaltungen eine hochkomplexe Handlung, da gleichzeitig verschiedene kommunikative Aufgaben, nämlich Hören und Schreiben, bewältigt werden müssen (Ehlich 2003, 19). Die für den erfolgreichen Wissenstransfer nötigen mental-rezeptiven und -produktiven Handlungen stellt Brinkschulte (2015) dar. So kann das Mitschreiben in Vorlesungen zum Lerngegenstand werden, um beispielsweise eigene Mitschriften zu analysieren und zu reflektieren, was von einer Vorlesung sinnvollerweise aufgeschrieben werden sollte, was nicht und wofür Mitschriften überhaupt eingesetzt werden können (Steets 2003, 61-62). Die Auseinandersetzung hierüber führt gleichzeitig zu einer vertieften Beschäftigung mit den Inhalten der Lehrveranstaltung sowie mit dem eigenen Lernverhalten.

Beim Zusammenfassen und Exzerpieren von Fachtexten wird geübt, wie Positionen und Aussagen von anderen neutral oder im Hinblick auf eine eigene Fragestellung sprachlich angemessen wiedergegeben werden können. Darüber hinaus wird erwartet, dass sich Studierende mit Inhalten kritisch auseinandersetzen und eine eigene Haltung in den Texten deutlich wird. Hierfür ist es notwendig, dass sich Studierende über ihre Rolle als Autor_innen bewusst werden und über sprachliche Mittel verfügen, Selbstreferenz zu formulieren (vgl. Kruse 2012; Steinhoff 2007a). Kruse $(2012,14)$ sieht in der »Konstruktion einer klaren Autorenrolle [...] eine große Herausforderung für Schreibende $\ll$. Diese Herausforderung zeigt sich beispielsweise in der Frage, ob »ich « verwendet werden darf oder nicht.

$\mathrm{Ob} \gg \mathrm{ich} \ll$ in einem wissenschaftlichen Text verwendet werden darf oder nicht, ist eine Frage, die unter Lehrenden und damit in den Disziplinen nicht einheitlich gehandhabt wird. Kretzenbacher $(1995,26)$ verwendete erstmals den Begriff »Ich-Tabu «, der seitdem durch die Wissenschaft geistert. Dabei zeigen korpuslinguistische Untersuchungen, dass der Gebrauch von »ich « (und » wir «) zwischen Disziplinen, Genres und Sprachen erheblich variiert (Kruse 2012, 14-15). Steinhoff (2007b) plädiert für einen differenzierten Ich-Gebrauch. Er differenziert zwischen der Verwendung eines Forscher_in-, Verfasser_in- und Erzähler_in-Ichs. Während die Forscher_in- und Verfasser_in-Ichs in wissenschaftlichen und akademischen Texten akzeptiert werden, wird das Erzähler_inIch in der Regel abgelehnt, da es autobiografische Informationen und subjektive Wertungen in den Text bringt, die von Wissenschaftler_innen, die diese Texte beurteilten sollten, als » nicht wissenschaftlich « angesehen werden (Steinhoff 2007b, 22-23).

Um für das eigene Schreiben Sicherheit im Umgang mit »ich « und der Konstruktion von Selbstreferenz zu erlangen, kann beispielsweise in Lehr-/Lernsituationen eine Auseinandersetzung mit der Ich-Typologie von Steinhoff erfolgen, um anschließend das erworbene Wissen auf Korpusbeispiele anzuwenden (vgl. Andresen und Knorr 2017) oder 
Formulierungsalternativen wie Verfahren der Deagentivierung zu erarbeiten. Auf diese Weise wird das theoretische Wissen über die Möglichkeiten zur Formulierung von Selbstreferenz vertieft und für den Umgang beim Schreiben mit »ich « sensibilisiert. Dabei ist anzumerken, dass die Verwendung von $\gg$ ich «-Formen nur eine von vielen Möglichkeiten der Selbstreferenz in akademischen Texten darstellt. Durch die Bewusstmachung, wie Autor_innen ihre Positionierung in ihre akademischen Texte einbringen und wie sie diese sprachlich ausdrücken können, erfahren sie einen Zugang zum Ausdruck ihres kritischen Denkens in ihrer Disziplin. Damit wird unter anderem mit der Fehlannahme von einigen Studienanfänger_innen aufgeräumt, dass sie ihre begründeten Positionierungen zu fachwissenschaftlichen Diskussionen in Texten nicht einbringen dürften.

\section{Den eigenen Schreibprozess produktiv steuern}

Kompetente Schreibende können ihren eigenen Schreibprozess produktiv steuern. Sie wählen also aus einem Repertoire an Schreibstrategien diejenige aus, mit der sie die gerade anstehende Aufgabe im Schreibprozess gut bewältigen können. Hierzu benötigen sie Wissen über die verschiedenen Anforderungsbereiche des Schreibens, wozu sprachliches Wissen, aber auch solches über Textorganisation und die Konstruktion der Autor_innenrolle sowie - nicht zuletzt - Wissen über den Schreibprozess selbst gehören (vgl. Baurmann und Pohl 2011; Becker-Mrotzek und Schindler 2007; Schindler und Siebert-Ott 2011).

Wissen über den Ablauf von Schreibprozessen, mögliche Schreibstrategien und die Auswirkungen des Einsatzes dieser Strategien auf das eigene Schreibhandeln kann einerseits durch Vermittlung erfolgen, andererseits sind eigene Erfahrungen notwendig. Die Vermittlung von Wissen über den Schreibprozess beinhaltet die Beschreibung von verschiedenen Phasen (vgl. Knorr 2016b) ebenso wie die der Funktionen des Schreibens für die Erkenntnisgewinnung (vgl. Kellogg 2008) sowie praktische Tipps zur Umsetzung (vgl. Bräuer, Brinkschulte und Halagan 2016). Der Aufbau metakognitiven Wissens beziehungsweise die Reflexion des eigenen Schreibhandelns unterstützt die Weiterentwicklung der eigenen Schreibkompetenz (vgl. Heine und Knorr 2017) sowie die einer fundierten Reflexionskompetenz (Bräuer, Brinkschulte und Halagan 2017).

\section{Den Textkonventionen der jeweiligen Fachgemeinschaft angemessen kommunizieren}

Wissen über Textarten ${ }^{2}$, Textmuster und fachliche Konventionen wird ebenfalls benötigt. Denn ein Protokoll eines chemischen Versuchs folgt anderen Vorgaben als eine 
Seminararbeit in der Erziehungswissenschaft. Und selbst ein und dieselbe Benennung eines Genres, wie beispielsweise Seminar- oder Hausarbeit, meint - wie Kruse und Chitez (2012) zeigen - in verschiedenen Fächern und an verschiedenen Standorten noch lange nicht dasselbe. Fachliche und kulturelle Einflüsse prägen die verschiedenen Textarten. Von Studierenden wird erwartet, dass sie im Laufe ihres Studiums die jeweiligen Anforderungen erkennen und sprachlich angemessen agieren können.

Studien aus verschiedenen Feldern der Sprachwissenschaft sowie aus anderen Disziplinen, wie zum Beispiel der Psychologie, Soziologie oder den Kulturwissenschaften, geben Aufschluss über den Aufbau von wissenschaftlichen Texten, deren Argumentationsstrukturen und die Art und Weise, wie sprachlich in diesen Texten gehandelt wird und wie Texte in Wissenschaftskulturen verankert sind sowie deren Weiterentwicklung mit prägen. Diskurstheoretische, sozio- und textlinguistische sowie funktional-pragmatische Ansätze liefern Erkenntnisse über das sprachliche Handeln in Texten und korpuslinguistische geben Aufschluss über die Sprachverwendung.

Benötigt wird hier jedoch noch weitere interdisziplinäre Forschung, die disziplinenspezifische und fachübergreifende Schreibprozesse, Charakteristika von Textkonventionen und wissenschaftssprachlichem Handeln aufdecken. Auch interkulturell angelegte Forschungen sind nötig, um zu sehen, wie Schreibende ihr bereits vorhandenes literales Wissen für das Schreiben in anderen Wissenschaftssprachen, Konventionen und Kontexten einbringen können. Das Ziel schreibwissenschaftlicher Forschung ist, sowohl Schreibprozesse als auch fachliche und sprachliche Konventionen sichtbar und damit vermittelbar zu machen. Denn eine immer wieder auftretende Schwierigkeit von Studierenden ist die implizite Wissensvermittlung fachlicher und sprachlicher Konventionen. Studierende - so zeigt es die Praxis - sind häufig unsicher ob ihres Sprachgebrauchs und der Umsetzung von Textkonventionen. Sie vermissen eine explizite Vermittlung von Schreibprozessen, die sie in verschiedenen Schreibarrangements üben können. Ob das Geschriebene » wissenschaftlich genug « sei oder wie ein Text wissenschaftlich aufgebaut sein müsse und wie ein Schreibprozess für einen akademischen Text organisiert sei, sind häufig gestellte Fragen in Schreibberatungen. Hierauf werden in Schreibberatungen Antworten erarbeitet, die zu den individuellen Haltungen, dem Vorwissen sowie zu den Anforderungen und der konkreten Schreibsituation passen, sodass der_die Schreibende sie für das Schreibprojekt umzusetzen weiß.

\section{Tätigkeitsfelder der Schreibzentrumsarbeit}

Schreibzentren und Schreibwerkstätten können als zentrale Einrichtungen oder als Teil einer Fakultät oder eines Instituts an Hochschulen verankert sein. Die Verankerung bestimmt zumeist die Art der Tätigkeit und die Zielgruppe, die angesprochen werden 
soll. Als zentrale Einrichtungen sind Schreibzentren in der Regel Ansprechpartner_in für die gesamte Hochschule; sind sie einzelnen Fakultäten oder Instituten zugeordnet, sind sie meist fachspezifisch ausgerichtet. Dementsprechend gibt es fachübergreifende Angebote oder solche, die in einem Fach verankert sind. Darüber hinaus nehmen einige Schreibzentren auch die sprachliche Diversität in den Blick, sodass hier zu den fachübergreifenden und fachnahen Angeboten noch ein weiterer Arbeitsschwerpunkt hinzukommt.

Neben der fachlichen Orientierung ist die Ausstattung mit Personal ein wesentlicher Faktor für die möglichen Tätigkeitsfelder von Schreibzentren. Knorr (2016a) gibt einen Überblick über die Ausstattung von Schreibberatungsprojekten, die über den Qualitätspakt Lehre finanziert werden: Einige Schreibberatungsprojekte bestehen aus einer halben wissenschaftlichen Mitarbeiter_innenstelle. Das Bielefelder Schreiblabor verfügt über neun Stellen und die Schreibwerkstatt Duisburg-Essen über 7,5 Stellen. Von den 20 Schreibprojekten, die aufgeführt werden, arbeiten drei Viertel mit SchreibPeer-Tutor_innen. Schreib-Peer-Tutor_innen sind Studierende, die schreibdidaktisch ausgebildet sind und auf Peer-Ebene mit Studierenden zusammenarbeiten, indem sie sie beraten und Lehrveranstaltungen übernehmen. Sie stärken damit das Peer-Learning und den Ansatz des Lernens durch Lehren. Gleichzeitig entwickeln sie durch ihre Tätigkeit ihre eigene Schreibkompetenz weiter.

In vielen Fällen war die Förderung studentischer Schreibfähigkeit ausschlaggebend für die Einrichtung eines Schreibzentrums. Die individuelle Beratung von Studierenden gehört daher zu den Standards. Allerdings ist dies nur ein Aspekt der Schreibzentrumsarbeit. Denn um studentische Schreibfähigkeit nachhaltig zu fördern, bedarf es der Unterstützung der Fachlehrenden. Gerade die Fachlehrenden sind diejenigen, die, neben den fachwissenschaftlichen Inhalten, das Schreiben als Lernfeld und als Medium zum kritischen Denken in ihre Lehre integrieren können. Schreibdidaktiker_innen unterstützen Fachlehrende dabei, das Schreiben in die Lehre zu integrieren und Konzepte schreibintensiver Lehre umzusetzen (Lahm 2016). Dabei sinkt im gelingenden Fall sogar die Arbeitsbelastung von Lehrenden und ermöglicht es Lehrenden, den eigenen Unterricht zu reflektieren. Dass hierfür jedoch Überzeugungsarbeit mit Geduld geleistet werden muss, zeigen vielfältige Berichte aus der Praxis der Schreibzentrumsarbeit. Darüber hinaus kooperieren Schreibzentrumsmitarbeitende mit anderen Einrichtungen der Hochschulen (bspw. Bibliotheken; vgl. Knorr 2019), um vorhandene Kompetenzen der Hochschulen zu verbinden, die beim wissenschaftlichen Schreiben benötigt werden. Auf diese Weise entsteht ein Kooperationsmodell, das am Beispiel des ersten Schreibzentrums in Deutschland, dem Schreiblabor Bielefeld ${ }^{3}$, illustriert werden soll. Das Schreiblabor Bielefeld wurde 1993 gegründet und ist mit seiner personellen Ausstattung eines der größten in Deutschland. Es ist an der Hochschule etabliert (inzwischen als Teil des Zentrums für Lehre und Lernen), sodass bei seiner Betrachtung 
auf Erfahrungswerte zurückgegriffen werden kann, die es bei vielen noch jüngeren Einrichtungen noch nicht geben kann.

Das Schreiblabor wendet sich an Studierende, Promovierende und Postdocs, Lehrende und Tutor_innen. Die Angebotspalette für Studierende reicht von Workshops über Einzel- und Gruppenberatungen, Vermittlung und Organisation von Schreibgruppen bis hin zur Veranstaltung von Schreibevents. Für Promovierende und Postdocs stehen individuelle Beratungen im Mittelpunkt.

Solche Angebote finden sich in ähnlicher Form in vielen anderen Schreibzentren. Zu den Workshopangeboten zählen solche über Schreib- und Lesestrategien und solche, die spezifische Herausforderungen des Schreibprozesses aufgreifen, wie beispielsweise das Überwinden von Hürden und Blockaden. Je nach Ausrichtung des Schreibzentrums können Angebote für bestimmte Zielgruppen innerhalb der Studierendenschaft hinzukommen. So gibt es beispielsweise an den Schreibzentren in Göttingen, Lüneburg und Tübingen Angebote für zweit- und fremdsprachig Schreibende oder auch die Thematisierung des akademischen Schreibens und Handelns in mehrsprachigen Kontexten für Studierende in internationalen Studiengängen. Die sprachenübergreifende Herangehensweise hat unseres Erachtens den besonderen Vorteil, dass zusätzlich zu fachübergreifenden und fachspezifischen Aspekten des Schreibens die Schreibenden auf sprachübergreifende Schreibstrategien zurückzugreifen lernen und das Potenzial mehrsprachigen Schreibens kennenlernen, zum Beispiel einen Text mehrsprachig zu planen, um sich auf die Inhalte zu konzentrieren und Spracharbeit sowie die Berücksichtigung von Textkonventionen anzuschließen.

Um das fachnahe Schreiben zu stärken, hat das Schreiblabor Bielefeld das Programm »Schreiben in den Fächern « entwickelt. Lehrende werden dazu angeregt, für sich zu explizieren, $\gg$ wie und warum sie als fachliche Expert/innen schreiben $\ll^{4}$. Ziel ist es, diese Erkenntnisse zu nutzen, um das Schreiben » als Lernmedium in der Lehre « einzubinden. Mitarbeiter_innen des Schreiblabors moderieren die Diskussion hierüber während eines monatlichen Jour fixe, bieten individuelle Beratungen und Kooperationen in Lehrveranstaltungen an.

Die Kooperationen in Lehrveranstaltungen sind schreibdidaktisch von besonderem Interesse. Schreibdidaktiker_innen entwickeln gemeinsam mit Fachlehrenden didaktische Umsetzungen schreibintensiver Lehre, sodass das Schreiben zum Lerngegenstand in der Fachlehre wird. Zudem wird ein weiterer zentraler Baustein der Schreibzentrumsarbeit integriert: das Peer-Learning. Hier werden zwei Formen unterschieden: die Ausbildung und schreibdidaktische Schulung von Studierenden zu studentischen Schreibberater_innen und die weitere Ausbildung dieser Studierenden zu »Writing Fellows «, sodass diese gezieltes Feedback auf Rohfassungen geben können.

Die schreibdidaktische Ausbildung von Studierenden ist nicht nur für das Bielefelder Schreibzentrum, sondern für viele Schreibzentren ein zentrales Anliegen. Im 
$\gg$ Rahmenkonzept für Ausbildungen von Peer-Schreibtutor*innen « (Special Interest Group »Qualitätsstandards und Inhalte der Peer-Schreibtutor*innen-Ausbildung « 2016) sind didaktische Grundlagen und Lernziele der Ausbildung erarbeitet worden, an denen sich immer mehr Schreibzentren orientieren. Das Verständnis der PeerSchreibberatung beruht auf dem Prinzip des Gesprächs auf Augenhöhe, in dem die studentischen Schreibberater_innen ihre Expertise für Texte und das Schreiben, die ratsuchenden Studierenden ihre für den Gegenstand mitbringen. Sie sind Peers, da es sich um Studierende handelt. Die Aufgabe von studentischen Schreibberater_innen ist es, ein professionelles Gespräch zu führen und die ratsuchenden Studierenden beim Finden von Lösungen zu unterstützen. Hierbei sollen sie überwiegend nicht instruierend vorgehen. Schreibberatung auf Peer-Ebene sei dabei lösungs- und ressourcenorientiert, personen- und kontextorientiert sowie ergebnisoffen (Special Interest Group »Qualitätsstandards und Inhalte der Peer-Schreibtutor*innen-Ausbildung « 2016, 2).

Writing Fellows sind $\gg$ studentische Schreibberater*innen, die schreibintensive Seminare an der Hochschule begleiten « (Voigt 2018, 15). Die Aufgaben der Writing Fellows besteht zum einen darin, Lehrenden ein Feedback aus ihrer studentischen Sicht auf Schreibaufgaben zu geben, die die Studierenden während des Semesterverlaufs bearbeiten. Zum anderen geben die Writing Fellows ein Feedback auf erste Versionen der Studierendentexte, sodass die Autor_innen ihre Texte zielorientiert überarbeiten können, bevor diese an die Lehrperson weitergereicht werden (Dreyfürst, Liebetanz und Voigt 2018). Writing Fellows nehmen eine Mittlerfunktion zwischen Studierenden und Lehrenden ein. Sie können gemeinsam mit den Lehrenden Erwartungen und Bewertungskriterien für wissenschaftliche Texte entwickeln und beim Design der Schreibaufgaben unterstützen (Voigt 2018, 15). Zu dieser Rollenspezifikation kommen Beschreibungen, was Writing Fellows nicht sind (Dreyfürst, Liebetanz und Voigt 2018, 3-4): Als Tutor_innen sind sie zwar keine Kursteilnehmende, aber sie sind selbst noch Studierende und damit keine Fachkraft in der Disziplin. Sie bieten kein Korrektorat und sollen weder von Studierenden noch von Lehrenden als Korrektor_innen in Anspruch genommen werden.

Da die Aufgaben von Schreib-Peer-Tutor_innen - Schreibberatungen durchzuführen, akademische Schreibprozesse zu vermitteln oder ein Textfeedback zu geben - sehr komplex sowohl inhaltlich als auch sozial äußerst anspruchsvoll sind, bedarf es einer fundierten Ausbildung, die Wissen über Erkenntnisse der Schreibprozessforschung und über Beratungsansätze, Kompetenzen in Didaktisierungen und für Schreibberatungen vermittelt, anwendet und übt. Ein wesentlicher Aspekt in der Ausbildung besteht darin, Rollen zu definieren und sich über die eigene Rolle im institutionellen Handeln bewusst zu werden. Dies ist eine Aufgabe, die in der Schreibberatungsausbildung intensiv behandelt werden muss, um Klarheit 
über Grenzen studentischer Tätigkeit als Schreibberater_in oder Writing Fellow zu gewinnen.

\section{Nutzen der Schreibzentrumsarbeit für die Hochschule}

Schreibzentren leisten einen Beitrag zur hochschuldidaktischen Entwicklung von Hochschulen. Über ihre schreibdidaktische Ausrichtung können sie mit allen Fächern, aber auch mit den verschiedenen Einrichtungen einer Hochschule ins Gespräch kommen, da sich ihr Blick auf eine Basiskompetenz wissenschaftlichen Arbeitens richtet. Ihre interdisziplinäre Ausrichtung ermöglicht eine Reflexion der Stellung und des Einsatzes von Schreibprozessen innerhalb von Fächern und das Herausarbeiten von fachspezifischen und fachübergreifenden Komponenten. Schreibzentrumsmitarbeiter_innen können solche Reflexionsprozesse initiieren und moderieren. Einmal sichtbar gemacht, können die Komponenten didaktisch aufbereitet und auf diese Weise lehrbar gemacht werden. Die Erfahrung von Schreibzentrumsmitarbeiter_innen in der Zusammenarbeit mit Fachlehrenden auf dieser Ebene zeigt, dass auf diese Weise alle Akteur_innen profitieren können: Im Vorfeld der Durchführung von Lehrveranstaltungen werden Fachlehrende durch die Kooperation mit Schreibzentrumsmitarbeiter_innen unterstützt, indem sie prüfen können, in welcher Form das Schreiben sinnvollerweise, also um das Lernziel der Veranstaltung zu erreichen, eingesetzt werden kann. In dieser Form leisten Schreibzentren einen hochschuldidaktischen Beitrag, um das Schreiben als Lerngegenstand in die Fachlehre zu integrieren. Notwendig sind hierfür vertrauensbildende Maßnahmen vonseiten der Schreibzentrumsmitarbeiter_innen gegenüber den Fachlehrenden: Schreibzentrumsmitarbeiter_innen bringen ihre Expertise hinsichtlich der Gestaltung von Schreibaufgaben ein, Fachlehrende entscheiden, inwieweit diese für die Erarbeitung von Fachinhalten geeignet sind. Auch wenn es aus schreibdidaktischer Perspektive sinnvoll wäre, Schreibkompetenzförderung in jedem Fach zu etablieren, ist schon aus personellen Gründen ein solcher Ansatz nicht durchführbar. Schreibzentrumsmitarbeiter_innen suchen daher an ihren Hochschulen Fachlehrende als Verbündete, die offen für schreibdidaktische Ideen und Konzepte sind. Dies sind in der Regel Fachlehrende, die ihre Lehre verändern möchten, weil sie mit den studentischen Texten unzufrieden sind. Diese kommen von sich aus auf das Schreibzentrum zu oder es ergeben sich Gesprächssituationen, aus denen Kooperationen entstehen. Die persönlichen Kontakte sind - so zeigt es der Erfahrungsaustausch unter Schreibzentrumsmitarbeiter_innen - zurzeit der am häufigsten gebrauchte Schlüssel zur Kooperation mit Fachlehrenden, da es an institutionellen Strukturen zur Förderung von Schreibkompetenz im Studium mangelt.

In der Arbeit mit Fachlehrenden können beispielsweise Schreibaufgaben besprochen und Anforderungen präzisiert und konkretisiert werden. Diese können dann 
wiederum den Studierenden vermittelt werden. Auf diese Weise entsteht Transparenz, die es Studierenden besser ermöglicht, die Schreibaufgaben zu bewältigen. Es wird aktiv gegen das sogenannte hidden curriculum gewirkt. Ein hidden curriculum meint die impliziten, also nicht formulierten Anforderungen, die Dozierende an die von Studierenden abzuliefernden Leistungen stellen. Die vorab erarbeitete Klarheit der Anforderungen erleichtert dem Lehrenden die Korrektur und Bewertung der schriftlichen Leistungen. Die Darlegung von Anforderungskriterien führt in der Regel zu einem besseren Verständnis der Studierenden für die gegebenen Noten.

Zudem unterstützen Schreibzentren Studierende durch ihre Angebote dabei, ihre Schreibkompetenz individuell zu entwickeln, indem sie selbst Lehrveranstaltungen zu verschiedenen Themen des akademischen Schreibens, Schreibwerkstätten, moderierte Schreibgruppen bis hin zu individuellen Schreibberatungen anbieten. Die meisten Schreibzentren setzen in der Arbeit mit den Studierenden ihre Schwerpunkte und eröffnen durch ein vielseitiges Programm, das die steigenden Ansprüche an das akademische Schreiben im Studienverlauf berücksichtigt, Studierenden die Möglichkeit, die Schlüsselkompetenz Schreiben auszubauen. Dies trägt maßgeblich zum Studienerfolg bei. Einige Schreibzentren, zum Beispiel Göttingen, bieten darüber hinaus eine gezielte Förderung beruflicher Schreibkompetenzen an. Vielfach sind die Angebote der Schreibzentren in den Schlüsselkompetenzen verankert.

\section{Ausblick}

Schreibzentrumsarbeit kann einen förderlichen Einfluss auf die hochschulische Bildungsqualität nehmen. Allerdings bedarf es hierfür struktureller und institutioneller Rahmenbedingungen, die erst an wenigen Hochschulen umgesetzt wurden. Wir, die Autorinnen dieses Beitrags, arbeiten an Hochschulen, an denen die Schreibzentrumsarbeit nachhaltig in die Struktur der Hochschule eingebunden wird. Dies zeigt sich daran, dass an der Leuphana Universität Lüneburg das Schreibzentrum/Writing Center vom Präsidium mit zwei unbefristeten Stellen sowie weiteren finanziellen und personellen Mitteln ausgestattet wurde. An der Georg-August-Universität Göttingen wurden vier Stellen entfristet sowie weitere personelle Maßnahmen und Sachmittel genehmigt, um das Internationale Schreibzentrum langfristig zu sichern. Für die allermeisten Schreibzentren und Schreibprojekte an deutschen Hochschulen gilt dies nicht. Sie sind über den Qualitätspakt Lehre finanziert, der 2020 endet. Die Diskussionen um die Weiterführung der Einrichtungen werden hochschulintern geführt. Allerdings ist die Aufgabe der akademischen Schreibausbildung von Studierenden nicht allein von den Hochschulen zu regeln; vielmehr handelt es sich um ein bildungspolitisches Anliegen, da der Bildungsauftrag von Hochschulen in einer Ausbildung von Studierenden zu Persön- 
lichkeiten liegt, die der Kraft sachlicher Argumente vertrauen und diese einzusetzen wissen (HRK 2018).

Der Aufgabe der nachhaltigen Etablierung von Schreibzentrumsarbeit an Hochschulen sowie der Etablierung von Begleitforschung der Schreibzentrumsarbeit widmet sich die Gesellschaft für Schreibdidaktik und Schreibforschung e.V., die international mit weiteren Gesellschaften zum akademischen Schreiben vernetzt ist. Die Schreibzentrumsarbeit steht im deutschsprachigen Hochschulkontext am Beginn ihrer Entwicklung, kann jedoch auf vielfältigen Erfahrungen und Forschungsergebnissen aus dem angloamerikanischen Raum aufbauen und verfügt über etablierte Konzepte, die durch Forschung zunehmend gesichert werden (vgl. bspw. Girgensohn 2017). Seit ihrem Start vor nunmehr 25 Jahren hat sie zentrale Schritte für die nachhaltige Etablierung von Schreibdidaktik sowie deren Erforschung an deutschsprachigen Hochschulen erreicht. Viele Fragen sind nach wie vor offen beziehungsweise werden bisher auf der Basis von Erfahrungswissen bearbeitet.

Die Förderung von Schreibkompetenzen ist unseres Erachtens eine gesamtuniversitäre Aufgabe, für die es weiterer Forschung und vor allem der Konzeptentwicklung bedarf, mit der auf die zunehmende sprachliche Heterogenität und bildungsbiografische Diversität der Studierenden reagiert werden kann. Rückmeldungen von Studierenden, Lehrenden, Studiendekanen und aus Präsidien von Hochschulen zeigen, dass die Arbeit von Schreibdidaktiker_innen zunehmend wertgeschätzt wird und als notwendiger Bestandteil für eine fundierte Ausbildung von Studierenden wahrgenommen wird. Allerdings fehlt es bislang an der nachhaltigen institutionellen Verankerung von Schreibzentren sowie schreibdidaktischer Tätigkeit an Hochschulen. Bislang fehlt es der Schreibdidaktik an empirisch fundierter Theoriebildung, die für die Etablierung des interdisziplinären Forschungsgegenstands Schreiben notwendig ist. Diese voranzubringen ist eine Aufgabe, der sich die schreibdidaktische Community gerne stellt beziehungsweise stellen möchte.

\section{Anmerkungen}

1 Die US-amerikanische Schreibausbildung, die bereits in den 1970er Jahren an Colleges und Universitäten weit verbreitet war, verfügt über eine breite Forschung zu schreibdidaktischen Handlungsfeldern. Emig (1971) kommt damals zu dem Schluss, dass ein überzeugender Text wenig über die Handlungen aussagt, die ein_e Schreiber_in durch- beziehungsweise ausgeführt hat, um diesen Text zu verfassen.

2 Zur theoretischen Fundierung und Differenzierung von Textart, Textsorte und Genre siehe Adamzik (2004).

3 http://uni-bielefeld.de/Universitaet/Einrichtungen/SLK/schreiblabor/(zugegriffen am 19.08.2018).

4 http://www.uni-bielefeld.de/Universitaet/Einrichtungen/SLK/schreiblabor/lehrende/ schreiben_in_den_faechern.html (zugegriffen am 19.08.2018). 


\section{Literatur}

Adamzik, Kirsten. 2004. Textlinguistik: Eine einführende Darstellung. Germanistische Arbeitshefte, Bd. 40. Tübingen: Niemeyer.

Alagöz-Bakan, Özlem, Dagmar Knorr und Kerstin Krüsemann, Hrsg. 2016. Akademisches Schreiben (Halbband 2). Sprache zum Schreiben - zum Denken - zum Beraten. Universitätskolleg-Schriften, $B d$. 14. Hamburg: Universität Hamburg.

Andresen, Melanie und Dagmar Knorr. 2017. „KoLaS. Ein Lernendenkorpus in der Schreibberatungsausbildung einsetzen«. Zeitschrift Schreiben 05.07.2017: 10-17. Zugegriffen am 08.09.2019. https://zeitschrift-schreiben.eu/globalassets/zeitschrift-schreiben.eu/2017/ andresen_knorr_lernendenkorpus-kolas2.pdf.

Ballweg, Sandra, Hrsg. 2016. Schreibberatung und Schreibförderung. Impulse aus Theorie, Emperie und Praxis. Wissen - Kompetenz - Text, Bd. 11. Frankfurt a. M. u. a.: Peter Lang.

Barczaitis, Irina, Melanie Brinkschulte und Ella Grieshammer. 2017. »MultiConText: Ein Konzept zur Förderung akademischer Schreibkompetenz für mehrsprachige Wissenschaftskontexte«. In Internationalisierung der Curricula an Hochschulen. Konzepte, Initiativen, Maßnahmen, hrsg. v. Hiltraud Casper-Hehne und Tanja Reiffenrath. Bielefeld: wbv.

Baurmann, Jürgen und Thorsten Pohl. 2011. „Schreiben - Texte verfassen«. In Bildungsstandards für die Grundschule, hrsg. v. Albert Bremerich-Vos, Dietlinde Granzer, Ulrike Behrens und Olaf Köller, 75-103. Berlin: Cornelsen.

Becker-Mrotzek, Michael, Joachim Grabowski und Thorsten Steinhoff, Hrsg. 2016. Forschungshandbuch empirische Schreibdidaktik. Münster: Waxmann.

Becker-Mrotzek, Michael und Kirsten Schindler. 2007. "Schreibkompetenzen modellieren«. In Texte schreiben, hrsg. v. Michael Becker-Mrotzek und Kirsten Schindler, 7-26. Duisburg: Gilles \& Francke.

Böhner, Marina, Nils Cordes, Denis Hedermann, Tina Hildebrand, Svenja Kaduk, Swantje Lahm, Elke Langelahn et al. 2016. »richtig einsteigen « mit literalen Kompetenzen an der Universität Bielefeld«. In Akademisches Schreiben (Halbband 1). Vom Qualitätspakt Lehre I geförderte Schreibprojekte, hrsg. v. Dagmar Knorr, 39-45. Hamburg: Universität Hamburg.

Bräuer, Christoph, Melanie Brinkschulte und Robert Halagan. 2016. »Akademisches Schreiben lernen lehren lernen«. In Akademisches Schreiben. Lehren und Lernen. Osnabrücker Beiträge zur Sprachtheorie, Bd. 88, hrsg. v. Christoph Bräuer und Melanie Brinkschulte, 89-119. Duisburg: Universitätsverlag Rhein Ruhr.

Bräuer, Christoph, Melanie Brinkschulte und Robert Halagan. 2017. „Strategien im akademischen Schreiben. Zum Potential von Schreibstrategien im Rahmen einer Unterrichtsreihe zu >Wissenschaftspropädeutik im Seminarfach an Gymnasien«. In Schreiben im Übergang von Bildungsinstitutionen, hrsg. v. Dagmar Knorr, Katrin Lehnen und Kirsten Schindler, 49-72. Frankfurt a. M.: Peter Lang.

Bräuer, Gerd. 2016. Das Portfolio als Reflexionsmedium für Lehrende und Studierende. Opladen \& Toronto: Budrich.

Brinkschulte, Melanie. 2012. »Akademisch Schreiben in der Fremd- und Zweitsprache Deutsch«. In Schreiben(d) lernen im Team. Ein Seminarkonzept für innovative Hochschullehre, hrsg. v. Kristin Draheim, Franziska Liebetanz und Stefanie Vogler-Lipp, 59-81. Wiesbaden: Springer VS.

Brinkschulte, Melanie. 2015. (Multi-)mediale Wissensübermittlung in universitären Vorlesungen. Eine diskursanalytische Untersuchung am Beispiel der Wirtschaftswissenschaft. Wissenschaftskommunikation, Bd. 11. Heidelberg: Synchron Wissenschaftsverlag der Autoren, Synchron Publishers.

Brinkschulte, Melanie. 2016. »Mehrsprachigkeit als Ressource für akademisches Schreiben«. In Schreibberatung und Schreibförderung. Impulse aus Theorie, Emperie und Praxis, hrsg. v. Sandra Ballweg, 97-114. Frankfurt a.M. u. a.: Peter Lang. 
Brinkschulte, Melanie und David Kreitz, Hrsg. 2017. Qualitative Methoden in der Schreibforschung. Bd. 1: Theorie und Praxis der Schreibwissenschaft. Bielefeld: wbv.

Deutsche Forschungsgemeinschaft. 2013. Sicherung guter wissenschaftlicher Praxis/Safeguarding Good Scientific Practice. Denkschrift/Memorandum. Erg. Aufl. Weinheim: Wiley-VCH.

Dreyfürst, Stephanie, Franziska Liebetanz und Anja Voigt. 2018. Das Writing Fellow-Programm. Ein Praxishandbuch zum Schreiben in der Lehre. Bd. 3: Theorie und Praxis der Schreibwissenschaft. Münster: Waxmann.

Ehlich, Konrad. 1999. »Alltägliche Wissenschaftssprache«. Info DaF 26 (1): 3-24.

Ehlich, Konrad. 2003. »Universitäre Textarten, universitäre Struktur«. In Wissenschaftlich Schreiben. Lehren und Lernen, hrsg. v. Konrad Ehlich und Angelika Steets, 13-28. Berlin \& New York: de Gruyter.

Emig, Janet. 1971. The Composing Processes of Twelfth Graders. Urbana: National Council of Teachers of English.

Flower, Linda S. und John R. Hayes. 1980. »The Dynamics of Composing. Making Plans and Juggling Constraints". In Cognitive Processes in Writing, hrsg. v. Lee W. Gregg und Erwin R. Steinberg, 31-50. Hillsdale, NJ: Erlbaum.

Gesellschaft für Schreibdidaktik und Schreibforschung. 2018. »Positionspapier Schreibkompetenz im Studium. Verabschiedet am 29. September 2018 in Nürnberg«. Zugegriffen am 08.09.2019. https://gefsus.de/images/Downloads/gefsus_2018_positionspapier.pdf.

Girgensohn, Kartin. 2017. Von der Innovation zur Institution. Institutionalisierungsarbeit an Hochschulen am Beispiel der Leitung von Schreibzentren. Bielefeld: Bertelsmann.

Graefen, Gabriele. 1997. Der wissenschaftliche Artikel. Textart und Textorganisation. Arbeiten zur Sprachanalyse, Bd. 27. Frankfurt a. M. u. a.: Peter Lang.

Hayes, John R. 2012. »Modeling and Remodeling Writing«. Written Communication 29 (3): 369-88. https://doi.org/10.1177/0741088312451260.

Hayes, John R. und Linda S. Flower. 1980. »Identifying the Organization of Writing Processes«. In Cognitive Processes in Writing, hrsg. v. Lee W. Gregg und Erwin R. Steinberg, 3-30. Hillsdale, NJ: Erlbaum.

Heine, Carmen und Dagmar Knorr. 2017. „Selbstreflexion akademischen Schreibhandelns anstoßen. Nicht-direktive Gesprächsführung als Haltung des Betreuenden«. In Schreiben im Übergang von Bildungsinstitutionen, hrsg. v. Dagmar Knorr, Katrin Lehnen und Kirsten Schindler, 109-131. Frankfurt a.M. u.a.: Peter Lang.

Jakobs, Eva-Maria. 1997. »Textproduktion als domänen- und kulturspezifisches Handeln. Diskutiert am Beispiel wissenschaftlichen Schreibens«. In Domänen- und kulturspezifisches Schreiben, hrsg. v. Kirsten Adamzik, Eva-Maria Jakobs und Gerd Antos, 9-30. Frankfurt a.M. u. a.: Peter Lang.

Jakobs, Eva-Maria. 1999. »Normen der Textgestaltung «. In Schlüsselkompetenz Schreiben. Konzepte, Methoden, Projekte für Schreibberatung und Schreibdidaktik an der Hochschule, hrsg. v. Otto Kruse, Eva-Maria Jakobs und Gabriela Ruhmann, 171-190. Neuwied \& Kriftel: Luchterhand.

Jakobs, Eva-Maria und Daniel Perrin, Hrsg. 2014. Handbook of Writing and Text Production. Berlin u. a.: de Gruyter.

Kellogg, Ronald T. 2008. »Training writing skills. A cognitive developmental perspective«. Journal of writing research 1 (1): 1-26.

Knorr, Dagmar. 2016a. »Einführung. Vom Qualitätspakt Lehre I geförderte Schreibprojekte«. In Akademisches Schreiben (Halbband 1). Vom Qualitätspakt Lehre I geförderte Schreibprojekte, hrsg. v. Dagmar Knorr, 11-24. Hamburg: Universität Hamburg.

Knorr, Dagmar. 2016b. »Modell `Phasen und Handlungen akademischer Textproduktion`. Eine Visualisierung zur Beschreibung von Textproduktionsprojekten«. In Schreibberatung und 
Schreibtraining. Impulse aus Theorie, Empirie und Praxis, hrsg. v. Sandra Ballweg, 251-73. Frankfurt a.M. u. a.: Peter Lang.

Knorr, Dagmar. 2019 »Schreibdidaktik und Hochschuleinrichtungen gemeinsam auf dem Weg zu einer akademischen Schreibkultur. Kooperationsmöglichkeiten von Hochschuleinrichtungen zur Förderung studentischen Schreibens«. In Praxishandbuch: Wissenschaftliches Schreiben in der Hochschulbibliothek, hrsg. v. Wilfried Sühl-Strohmenger und Ladina Tschander, 46-56. Berlin: de Gruyter.

Knorr, Dagmar, Carmen Heine und Jan Engberg, Hrsg. 2014. Methods in writing process research. Textproduktion und Medium, Bd. 13. Frankfurt a. M. u. a.: Peter Lang.

Knorr, Dagmar, Melanie Andresen, Özlem Alagöz-Bakan und Anna Tilmans. 2015. »Mehrsprachigkeit. Ressource für SchreibberaterInnen und Ratsuchende«. In Impulse für die Migrationsgesellschaft. Bildung, Politik und Religion, hrsg. v. Inci Dirim, Ingrid Gogolin, Dagmar Knorr, Marianne Krüger-Potratz, Drorit Lengyel, Hans H. Reich und Wolfram Weiße, 318-38. Münster u.a.: Waxmann.

Kretzenbacher, Heinz Leonhard. 1995. „Wie durchsichtig ist die Sprache der Wissenschaften?«. In Linguistik der Wissenschaftssprache, hrsg. v. Heinz Leonhard Kretzenbacher und Harald Weinrich, 15-39. Berlin \& New York: de Gruyter.

Krings, Hans P. 1992. »Schwarze Spuren auf weißem Grund. Fragen, Methoden und Ergebnisse der Schreibprozeßforschung im Überblick«. In Textproduktion. Neue Wege der Forschung, hrsg. v. Hans P. Krings und Gerd Antos, 45-110. Trier: Wissenschaftlicher Verlag Trier.

Kruse, Otto. 2007. "Schreibkompetenz und Studierfähigkeit: Mit welchen Schreibkompetenzen sollten die Schulen ihre Absolvent/innen ins Studium entlassen?«. In Texte schreiben, hrsg. v. Michael Becker-Mrotzek und Kirsten Schindler, 117-143. Duisburg: Gilles \& Francke.

Kruse, Otto. 2012. »Wissenschaftliches Schreiben mehrsprachig unterrichten. Was ist möglich, was ist nötig?«. ÖDaF-Mitteilungen 28 (2): 9-25.

Kruse, Otto. 2017. Kritisches Denken und Argumentieren. Studieren, aber richtig, Bd. 4767. Konstanz: Huter \& Roth KG, UVK Verlagsgesellschaft mbH.

Kruse, Otto und Eva-Maria Jakobs. 1999. »Schreiben lehren an der Hochschule. Ein Überblick«. In Schlüsselkompetenz Schreiben. Konzepte, Methoden, Projekte für Schreibberatung und Schreibdidaktik an der Hochschule, hrsg. v. Otto Kruse, Eva-Maria Jakobs und Gabriela Ruhmann, 19-34. Neuwied, Krifke \& Berlin: Luchterhand.

Kruse, Otto und Gabriela Ruhmann. 2006. »Prozessorientierte Schreibdidaktik. Eine Einführung «. In Prozessorientierte Schreibdidaktik. Schreibtraining für Schule, Studium und Beruf, hrsg. v. Otto Kruse, Eva Berger und Marianne Ulmi, 13-35. Bern, Stuttgart \& Wien: Haupt.

Kruse, Otto und Madaline Chitez. 2012. »Kontrastives Genre-Mapping. Didaktische Genres an Pädagogischen Hochschulen des französisch-, deutsch- und italienischsprachigen Teils der Schweiz«. In Schreiben unter Bedingungen von Mehrsprachigkeit, hrsg. v. Dagmar Knorr und Annette Verhein-Jarren, 9-34. Frankfurt a.M. u. a.: Peter Lang.

Kultusministerkonferenz. 2017.»Qualifikationsrahmen für deutsche Hochschulabschlüsse (Im Zusammenwirken von Hochschulrektorenkonferenz und Kultusministerkonferenzund in Abstimmung mit Bundesministerium für Bildung und Forschung erarbeitet und von der Kultusministerkonferenzam 16.02.2017 beschlossen)«.Zugegriffen am 08.09.2019. https://www.kmk.org/fileadmin/ Dateien/veroeffentlichungen_beschluesse/2017/2017_02_16-Qualifikationsrahmen.pdf.

Lahm, Swantje. 2016. Schreiben in der Lehre. Opladen \& Toronto: Budrich.

Lange, Ulrike. 2015. »Mehrsprachige Wege zu einsprachigen Texten. Eine Unterrichtsreihe mit Schreibstationen«. ide. Informationen zur Deutschdidaktik 39 (4): 133-41.

Pohl, Thorsten und Torsten Steinhoff. 2010. »Textformen als Lernformen«. In Textformen als Lernformen, hrsg. v. Thorsten Pohl und Torsten Steinhoff, 5-26. Köln: Gilles \& Francke. 
Portmann-Tselikas, Paul R. 1991. Schreiben und Lernen. Grundlagen der fremdsprachlichen Schreibdidaktik. Germanistische Linguistik, Bd. 122. Tübingen: Niemeyer.

Schindler, Kirsten und Gesa Siebert-Ott. 2011. »Entwicklung der Textkompetenz von Studierenden (in der Zweitsprache Deutsch) - Propädeutik, akademisches und berufsbezogenes Schreiben«. In Sprachliche Förderung und Weiterbildung - transdisziplinär, hrsg. v. Andreas Krafft und Carmen Spiegel, 91-110. Frankfurt a. M.: Peter Lang.

Senat der Hochschulrektorenkonferenz (HRK). 2018. "Die Hochschulen als zentrale Akteure in Wissenschaft und Gesellschaft. Eckpunkte zur Rolle und zu den Herausforderungen des Hochschulsystems«. Zugegriffen am 08.09.2019. https://www.hrk.de/fileadmin/redaktion/ hrk/02-Dokumente/02-01-Beschluesse/HRK_-_Eckpunkte_HS-System_2018.pdf.

Special Interest Group »Qualitätsstandards und Inhalte der Peer-Schreibtutor*innen-Ausbildung«. 2016. »Rahmenkonzept für Ausbildungen von Peer-Schreibtutor*innen«, hrsg. v. der Gesellschaft für Schreibdidaktik und Schreibforschung. Zugegriffen am 08.09.2019. https://gefsus. de/images/Downloads/RahmenkonzepPeerSchreibtutor_innenausbildg.pdf.

Steets, Angelika. 2003. »Die Mitschrift als universitäre Textart. Schwieriger als gedacht, wichtiger als vermutet«. In Wissenschaftlich schreiben. Lehren und lernen, hrsg. v. Konrad Ehlich und Angelika Steets, 51-64. Berlin: de Gruyter.

Steinhoff, Torsten. 2007a. Wissenschaftliche Textkompetenz. Sprachgebrauch und Schreibentwicklung in wissenschaftlichen Texten von Studenten und Experten. Germanistische Linguistik, Bd. 280. Tübingen: Niemeyer.

Steinhoff, Torsten. 2007b. »Zum ich-Gebrauch in Wissenschaftstexten«. ZGL 35 (2): 1-26. https:// doi.org/10.1515/ZGL.2007.002.

Stezano Cotelo, Kristin. 2011. »Aufgabenbewältigung. Der Weg zum wissenschaftlichen Schreiben am Beispiel von Seminararbeiten fremdsprachlicher Studierender «. In Fremdsprachliche Textkompetenz entwickeln, hrsg. v. Dagmar Knorr und Antonella Nardi, 91-110. Frankfurt a.M. u. a.: Peter Lang.

Voigt, Anja, Hrsg. 2018. Lehren und Lernen mit Writing Fellows. Beiträge zur Forschung, Evaluation und Adaption. Theorie und Praxis der Schreibwissenschaft, Bd. 4. Münster: Waxmann.

\section{Die Autorinnen}

Dagmar Knorr, Dr., Leiterin des Schreibzentrums/Writing Center der Leuphana Universität Lüneburg. Arbeitsschwerpunkte: Akademische Schreibdidaktik und Schreibforschung, Schreiben unter Bedingungen von Mehrsprachigkeit und vor dem Hintergrund heterogener Bildungsbiografien, Schreibkompetenzentwicklung im Studium, Evaluation von Schreibzentrumsarbeit.

Kontakt: dagmar.knorr@leuphana.de

Melanie Brinkschulte, Dr., Leiterin des Schlüsselkompetenzbereichs »Interkulturelle Interaktionen«: Internationales Schreibzentrum, Deutsch als Fremdsprache, Interkulturelles Kompetenzzentrum. Arbeitsschwerpunkte: Sprachlehr- und -lernforschung, Akademische Schreibdidaktik und Schreibforschung, Didaktik für akademisches Schreiben in mehrsprachigen Kontexten, Ausbildung von Schreibberater_innen, Qualitätssicherung in der Schreibzentrumsarbeit, kollaborative Lehr- und Lernformen zur Förderung von Reflexionskompetenzen.

Kontakt: melanie.brinkschulte@phil.uni-goettingen.de 